\title{
Gas-Mediated Electron Beam Induced Etching - From Fundamental Physics to Device Fabrication.
}

\author{
A. A. Martin ${ }^{1}$, I. Aharonovich ${ }^{1}$ and M. Toth $^{1}$ \\ 1. School of Physics and Advanced Materials, University of Technology, Sydney, Australia.
}

Gas-mediated electron beam induced etching (EBIE) is a nanoscale, direct-write technique analogous to gas-assisted focused ion beam (FIB) milling. The main advantage of EBIE is the elimination of sputtering and ion implantation during processing as well as greater material selectivity [1]. Here we discuss recent developments that expand the scope of EBIE applications in nanofabrication and defect generation analysis, and show advances in hardware that open the door for new studies in reaction kinetics using a scanning electron microscope (SEM).

First, we demonstrate EBIE as a tool to probe the damage generation kinetics of materials under low energy electron irradiation [2]. Ultra nanocrystalline diamond (UNCD) initially exhibits negligible etching ascribed to a low volatilization rate of $s p^{3}$ rich carbon by reactive fragments produced through electron induced dissociation of $\mathrm{H}_{2} \mathrm{O}$ adsorbates. Etching of UNCD accelerates significantly upon irradiation by low energy $(5-20 \mathrm{keV}$ ) electrons, and the rate scales inversely with electron beam energy and directly with energy density deposited into the solid. By incorporating the dependence of EBIE efficiency on electron-restructuring of a substrate into existing etch kinetics models (Figure 1a), we can form an understanding of electron-solid interaction within the material. For the case of UNCD, EBIE is shown to proceed through an electron-restructuring pathway that generates defect rich carbon at the nanosized grain boundaries that is susceptible to volatilization.

Second, we present a novel way to fabricate diamond nanostructures in single crystal diamond with $\sim 100$ $\mathrm{nm}$ nanometer resolution using EBIE. Diamond has found applications across numerous areas of science due to its unique combination of optical, electronic, chemical and thermal properties. However, before diamond can transform from the laboratory setting to a practical device, robust fabrication methods must be developed. $\mathrm{H}_{2} \mathrm{O}$-mediated EBIE is employed in our case for mask-free editing of inclined diamond surfaces (Figure 1b). Editing of multiple inclined facets is nearly impossible by mask based processing techniques, including electron- and photo- lithography. Fabricated structures, including pillar cavities and diamond nanostructures retain their optical properties and exhibit bright florescence. The advanced functionalities provided by a conventional SEM in conjunction with EBIE will enable the modification to existing devices and direct nanofabrication for rapid prototyping with diamond.

Finally we discuss hardware advances that give rise to new areas of EBIE research. Cryogenic cooling is used to enable efficient EBIE in systems where etching is negligible at elevated substrate temperatures due to weak physisorption of precursor molecules to the substrate. It is demonstrated using $\mathrm{NF}_{3}$ precursor gas, and $\mathrm{Si}, \mathrm{SiC}, \mathrm{SiO}_{2}$, and $\mathrm{Si}_{3} \mathrm{~N}_{4}$ substrates (Figure 2a). This technique enables high resolution EBIE in the absence of artifacts caused by delocalized spontaneous etching of the substrates. Furthermore development of an environmental reaction cell sub-chamber (Figure $2 b$ ) is discussed as a tool in applications where residual $\mathrm{H}_{2} \mathrm{O}$ affects reaction kinetics and for in situ analysis of chemical vapor deposition (CVD) processes in a SEM [3]. The reaction cell is constructed from ultra high vacuum components allowing for high temperature operation and removal of surface contaminants, and 
the inclusion of a gaseous secondary electron detector allows for imaging under high pressure gas environments.

The versatility and the flexibility of EBIE as a tool for probing electron-solid-gas interactions is now expanding. As this knowledge increases new nanofabrication processes using EBIE are being developed for current silicon-based and future optoelectronic devices [4].

\section{References:}

[1] "Nanofabrication Using Focused Ion and Electron Beams: Principles and Applications", ed. I. Utke, S. Moshkalev, and P. Russell, (Oxford University Press, New York).

[2] A. A. Martin, M. Phillips, and M. Toth, ACS Appl. Mater. Interfaces 5 (2013), p. 8002-8007.

[3] C. J. Lobo, A. A. Martin, M. R. Phillips, and M. Toth, Nanotechnology 23 (2012), p. 375302.

[4] Acknowledgments: This work was partly funded by FEI Company. A. Martin is the recipient of a John Stocker Postgraduate Scholarship from the Science and Industry Endowment Fund. I. Aharonovich is the recipient of an Australian Research Council Discovery Early Career Research Award (Project Number DE130100592).
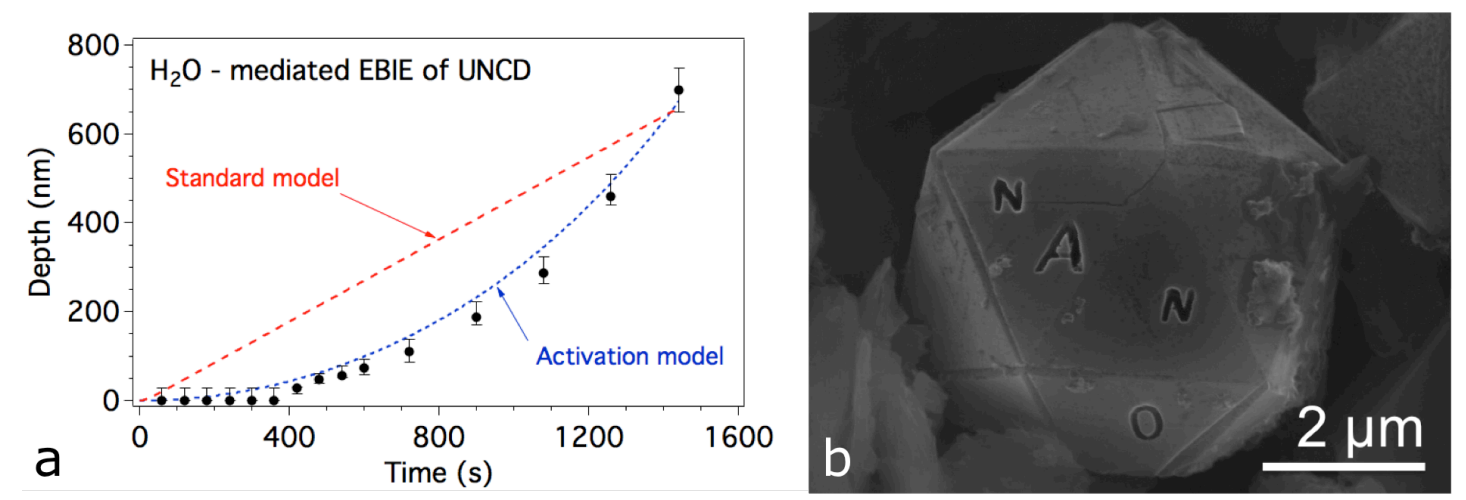

Figure 1. a) Experimental etch pit depth versus time for $\mathrm{H}_{2} \mathrm{O}$-mediated EBIE of UNCD, and calculated etch profiles using the standard and dynamic surface site activation EBIE models. b) "NANO" written on three separate diamond micro-particle (111) faces, showing the power of EBIE for nanofabrication.
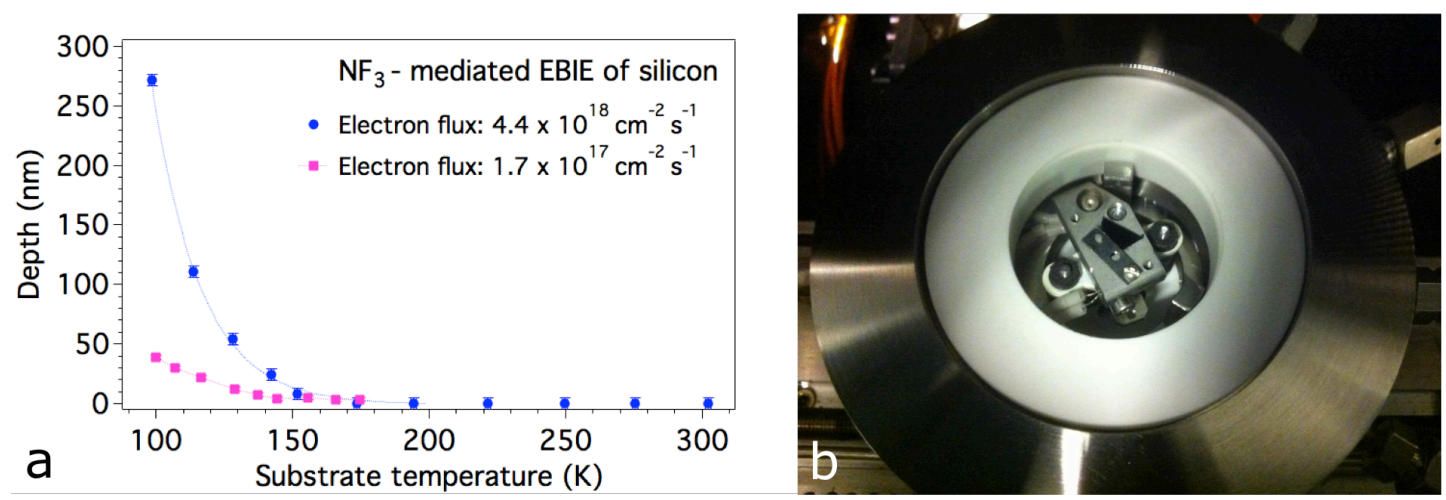

Figure 2. a) Experimental etch pit depth versus substrate temperature for NF3-mediated EBIE of silicon. b) Environmental reaction cell sub-chamber used for high purity EBIE and CVD studies in a SEM. 